\title{
Habitable planet formation in extreme planetary systems: systems with multiple stars and/or multiple planets
}

\author{
Nader Haghighipour \\ Institute for Astronomy and NASA Astrobiology Institute, \\ University of Hawaii-Manoa, Honolulu, HI
}

\begin{abstract}
Understanding the formation and dynamical evolution of habitable planets in extrasolar planetary systems is a challenging task. In this respect, systems with multiple giant planets and/or multiple stars present special complications. The formation of habitable planets in these environments is strongly affected by the dynamics of their giant planets and/or their stellar companions. These objects have profound effects on the structure of the disk of planetesimals and protoplanetary objects in which terrestrial-class planets are formed. To what extent the current theories of planet formation can be applied to such "extreme" planetary systems depends on the dynamical characteristics of their planets and/or their binary stars. In this paper, I present the results of a study of the possibility of the existence of Earth-like objects in systems with multiple giant planets (namely $v$ Andromedae, $47 \mathrm{UMa}$, GJ 876, and $55 \mathrm{Cnc}$ ) and discuss the dynamics of the newly discovered Neptune-sized object in 55 Cnc system. I will also review habitable planet formation in binary systems and present the results of a systematic search of the parameter-space for which Earth-like objects can form and maintain long-term stable orbits in the habitable zones of binary stars.
\end{abstract}

Keywords. (stars:) planetary systems: formation, celestial mechanics, methods: numerical

\section{Introduction}

The discovery of extrasolar planets during the past decade has confronted astronomers with many new challenges. The diverse and surprising dynamical characteristics of many of these objects have made scientists wonder to what extent the current theories of planet formation can be applied to other planetary systems. A major challenge of planetary science is now to explain how such planets were formed, how they acquired their unfamiliar dynamical state, and whether they can be habitable.

Among the unfamiliar characteristics of the currently known extrasolar planetary systems, the existence of systems with multiple planets in which Jovian-type bodies are in eccentric and close-in orbits, and the existence of Jupiter-like planets in multi-star systems are particularly interesting. As shown in figure 1, at the present, 26 extrasolar planetary systems contain more than one giant planet. Also, as shown in Table 1, more than $20 \%$ of planet-hosting stars are members of binary systems. The formation of terrestrial-class objects in such planetary systems, and the possibility of their long-term stability in the habitable zones of their host stars are strongly affected by the dynamical perturbations of the giant planets, and in systems of binaries with separations smaller than $100 \mathrm{AU}$, by the perturbation of the stellar companion. Whether such "extreme" planetary environments can be potential hosts to habitable planets is the subject of this paper. I will review the possibility of the long-term stability of terrestrial-class objects in some of multi-planet systems, and review the current status of research on planet formation in dual-star environments. 


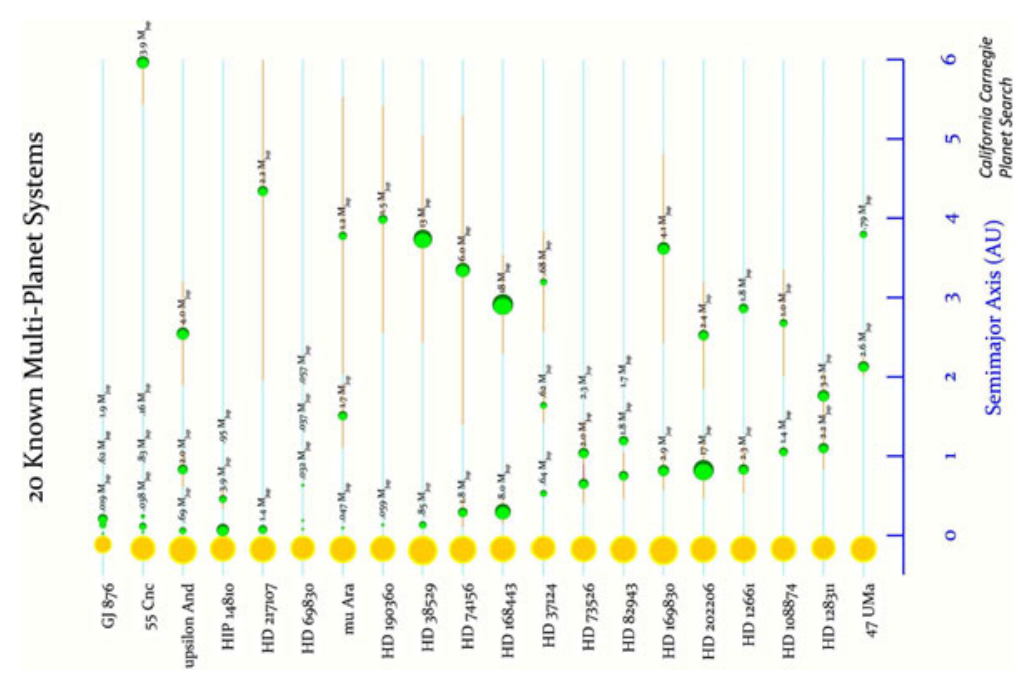

Figure 1. Currently known multi-planet extrasolar planetary systems (California-Carnegie Planet Search).

\section{Habitability of Extrasolar Multi-planet Systems}

In order for a planetary system to be habitable, an Earth-like planet has to maintain its orbit in the habitable zone (HZ) of the system's central star for a long time. This condition requires that the orbital eccentricity of a habitable planet to be close to zero and its interactions with other bodies of the system do not disturb its long-term stability. In a multi-planet system, these conditions may not be easily satisfied. The dynamics of an object in such systems is strongly affected by other planets and the habitability of a terrestrial planet may be influenced by the perturbations from giant bodies. The latter is more significant in systems where the orbits of giant planets are close to the habitable zone. The planetary systems of $v$ Andromedae $(\mathrm{HZ}=1.68-2 \mathrm{AU}), 47 \mathrm{UMa}(\mathrm{HZ}=1.16-$ 1.41 AU), GJ $876(\mathrm{HZ}=0.1-0.13 \mathrm{AU})$, and 55 Cancri $(\mathrm{HZ}=0.72-0.87 \mathrm{AU})$ are of this kind. In a recent article (Rivera \& Haghighipour, 2007), we studied the stability of terrestrial-class objects in these systems by numerically integrating the orbits of several hundred test particles, uniformly distributed along the $x$-axis, in initial circular orbits. Figure 2 shows the graphs of the lifetimes of these particles for $10 \mathrm{Myr}$. As shown here, unlike the stable orbit of the newly discovered Earth-like planet of GJ 876 (Rivera et al., 2005), the orbit of the small close-in planet of 55 Cnc, as reported by McArthur et al. (2004) is unstable. Our results also indicate that it is unlikely that $v$ Andromedae and GJ 876 harbor habitable planets. This has also been confirmed by the direct integration of the orbit of an Earth-sized object in the habitable zone of the system by Dove \& Haghighipour (2006). The two systems of $47 \mathrm{UMa}$ and $55 \mathrm{Cnc}$, however, have stable habitable zones, although direct integrations of actual Earth-like objects in these systems are necessary to confirm their habitability. The results of our test particle simulations also indicated the capability of $55 \mathrm{Cnc}$ system in harboring stable planet(s) in the region between 0.7 AU and 2.2 AU. As shown by Fischer et al. (2007) and as in figure 2, the newly discovered Neptune-sized planet of this system is located in this region. 
Table 1. Extrasolar Planet-Hosting Stars in Binary Systems (Haghighipour 2006)

\begin{tabular}{l|l|l|l}
\hline Star & Star & Star & Star \\
\hline HD142 (GJ 9002) & HD3651 & HD9826 $(v$ And $)$ & HD13445 (GJ 86) \\
HD19994 & HD22049 $(\epsilon$ Eri) & HD27442 & HD40979 \\
HD41004 & HD75732 $(55$ Cnc $)$ & HD 80606 & HD 89744 \\
HD114762 & HD117176 $(70$ Vir $)$ & HD120136 $(\tau$ Boo $)$ & HD121504 \\
HD137759 & HD143761 $(\rho$ Crb) & HD178911 & HD186472 (16 Cyg) \\
HD190360 (GJ 777A) & HD192263 & HD195019 & HD213240 \\
HD217107 & HD219449 & HD219542 & HD222404 $(\gamma$ Cephei) \\
HD178911 & HD202206 & PSR B1257-20 & PSR B1620-26 \\
\hline
\end{tabular}

\section{Habitability of Multiple Star Systems}

As shown in Table 1, more than $20 \%$ of currently known planet-hosting stars are members of binary systems (Haghighipour 2006). Many of these systems are wide with separations ranging from $200 \mathrm{AU}$ to $6000 \mathrm{AU}$. In such systems, the perturbative effect of the stellar companion is negligible and planet formation around the other star may proceed in the similar fashion as around a single star. There are, however, three binary systems, namely, GL 86 (Els et al. 2001), $\gamma$ Cephei (Hatzes et al. 2003), and HD 41004 (Zucker et al. 2004, Raghavan et al. 2006), in which the primary star is host to a Joviantype planet and the binary separation is smaller than 20 AU. How these planets were formed, and whether such binary-planetary systems can be habitable are now among major theoretical challenges of planetary dynamics.

Planet formation in close binary systems is strongly affected by the perturbation of the binary companion. This star may remove planet-forming material by truncating the primary's circumstellar disk (Artymowicz \& Lubow 1994) and destabilizing the regions where planetesimals and protoplanets may undergo collisional growth (Thébault et al. 2004). In binary systems where the primary hosts a giant planet, the perturbative effect of the planetary companion will also affect the growth of protoplanetary objects. However, as shown by numerical integrations of the orbits of Earth-sized planets in $\gamma$ Cephei system (Haghighipour 2006), it is possible for a terrestrial-class body to maintain a long-term stable orbit at distances close to the primary star and outside the giant planet's influence zone. Figure 3 shows the graph of the lifetime of an Earth-sized object in the system of $\gamma$ Cephei. As shown here, the HZ of the system is unstable. However, an Earth-like planet can main a stable orbit close to the primary star.

Based on the results of the simulations shown in figure 3, we recently studied habitable planet formation in moderately closed binary star systems that host giant planets (Haghighipour \& Raymond 2007). We simulated the late stage of terrestrial planet formation for different values of the semimajor axis and orbital eccentricity of the binary, as well as different binary mass-ratios. Our system consisted of a Sun-like star as the primary, a disk of protoplanetary bodies with 120 Moon- to Mars-sized objects distributed randomly between $0.5 \mathrm{AU}$ and $4 \mathrm{AU}$, and a Jupiter-sized planet at $5 \mathrm{AU}$. To study the effect of the orbital dynamics of the secondary star on the formation and water contents of planets in the $\mathrm{HZ}$ of the primary, we considered the orbit of the giant planet to be circular. We also assumed that, following the model of Morbidelli et al. (2000), in which the main source of Earth's water is the water-rich bodies in the solar system's asteroid belt, the water in the habitable planet of a binary system is originated from the asteroid region of its primary and is delivered to it during the collisional growth of the system's protoplanetary objects. We considered an initial gradient in the water contents of em- 

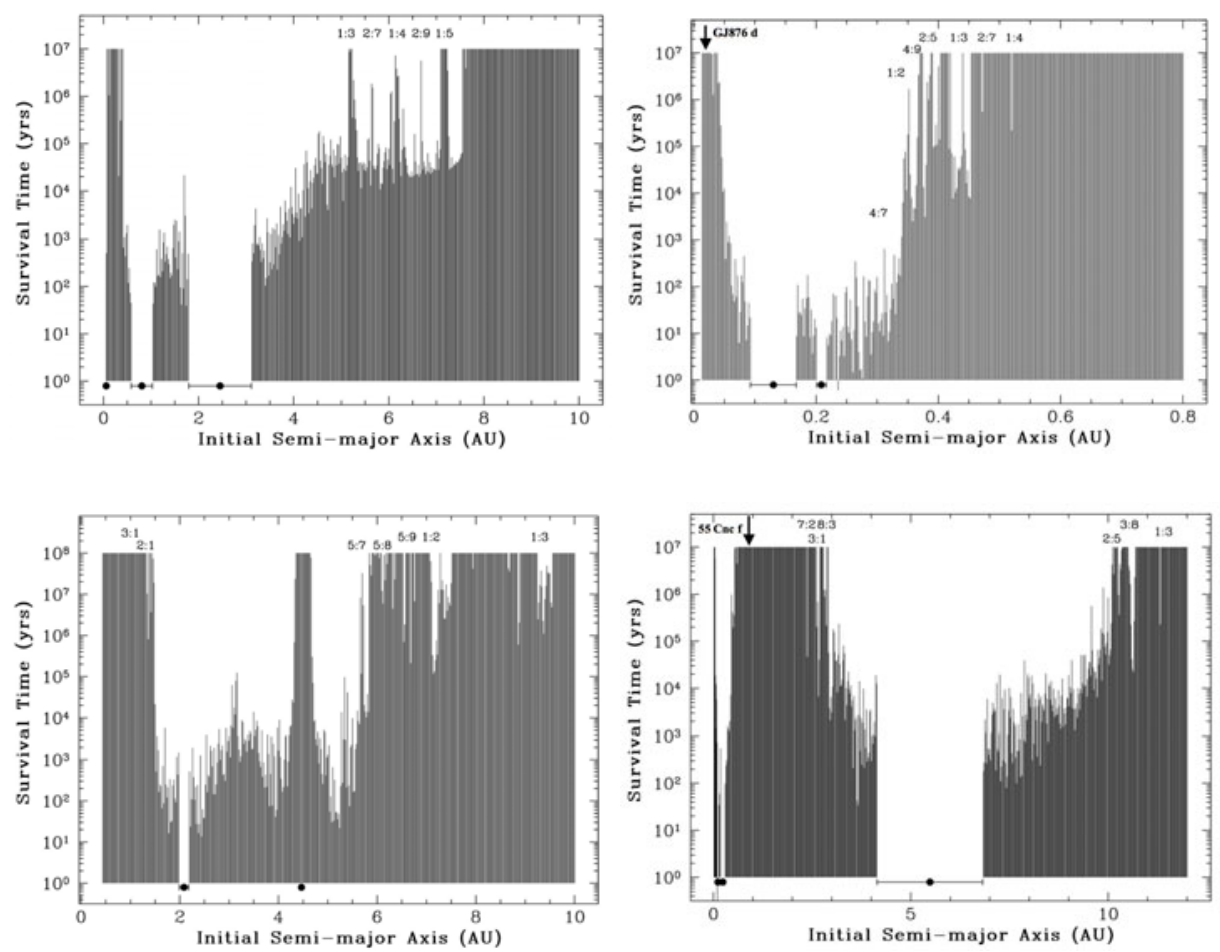

Figure 2. Graphs of the lifetimes of test particles in $v$ Andromedae (top left, $\mathrm{HZ}=1.68-2 \mathrm{AU}$ ), GJ 876 (top right, $\mathrm{HZ}=0.1-0.3 \mathrm{AU}$ ), $47 \mathrm{UMa}$ (bottom left, $\mathrm{HZ}=1.16-1.41$ ), and $55 \mathrm{Cnc}$ (bottom right, $0.72-0.87 \mathrm{AU}$ ). The graphs and habitable zones are from Rivera \& Haghighipour (2007). The islands of stability and instability, with their corresponding mean-motion resonances with the inner and/or outer planet are also shown. As shown here, the habitable zones of $v$ Andromedae and GJ 876 are unstable implying that the planetary systems of these stars will not be habitable. The habitable zones of $47 \mathrm{UMa}$ and $55 \mathrm{Cnc}$, on the other hand, are stable. Also as shown here, the recently detected Earth-like planet of GJ 876 (Rivera et al. 2005), and the newly discovered fifth planet of 55 Cnc (Fischer et al. 2007) are in stable orbits.

bryos similar to the distribution of water in the primitive asteroids of the asteroid belt (Abe et al. 2000). That is, embryos inside 2 AU were taken to be dry, the ones between 2 to $2.5 \mathrm{AU}$ were considered to contain $1 \%$ water, and those beyond $2.5 \mathrm{AU}$ were assumed to have a water to mass ratio of $5 \%$. Figure 4 shows some of the results for binary mass-ratios $\mu_{b}=0.5,1.5$. As shown here, it is possible to form Earth-like objects with substantial amount of water in the HZ of the primary star. The sizes of these planets and their water contents vary with the semimajor axis and eccentricity of the stellar companion. In binaries where the secondary star has a small periastron, the interaction between this object and the giant planet of the system, which transfers angular momentum to the disk of planetary embryos, causes many of these bodies to be ejected from the system. As a result, in closer and eccentric binaries, the final planets are smaller and contain less or no water. Figure 5 shows the relation between the periastron of the binary $\left(q_{b}\right)$ and the semimajor axis of the outermost terrestrial planet $\left(a_{o u t}\right)$. As shown in the left graph of figure 5, similar to Quintana et al. (2007), simulations with no giant planets favor 


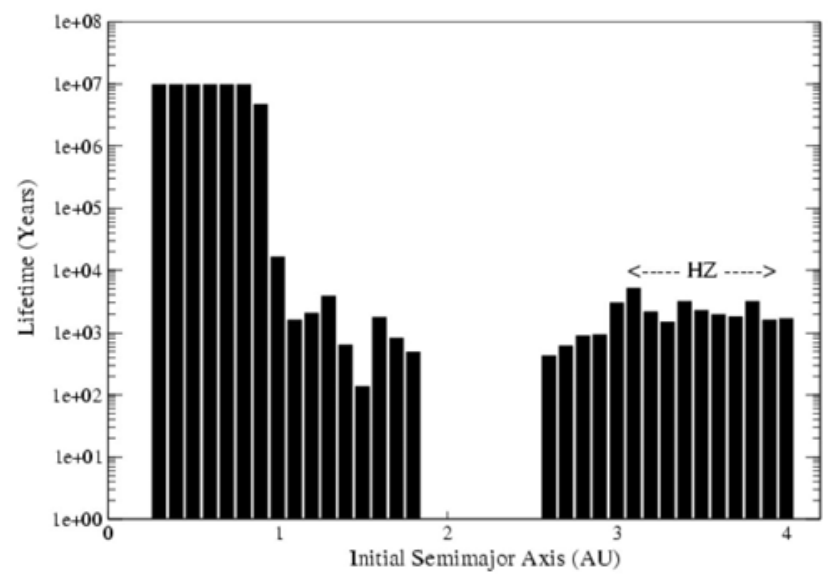

Figure 3. Lifetime of an Earth-sized planet in $\gamma$ Cephei system. The giant planet of the system (1.67 Jupiter-mass) is at $2.13 \mathrm{AU}$ with an eccentricity of 0.12 . As shown here, the HZ of the system is unstable. However, a terrestrial-class object can maintain a long-term orbit at close distances to the primary star (Haghighipour 2006).

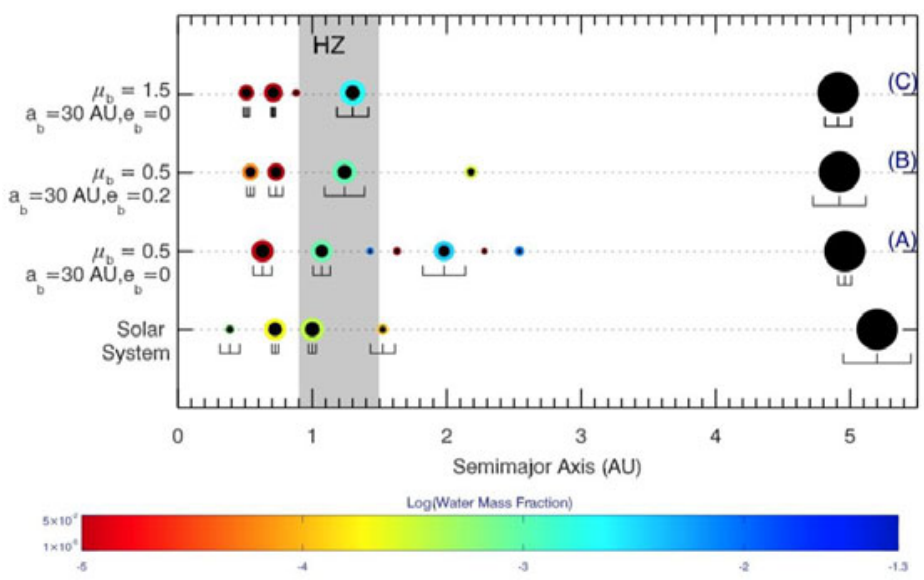

Figure 4. Habitable planet formation in binary-planetary systems with 0.5 and 1.5 stellar mass-ratios. As shown here, binaries with moderate periastron distances are more favorable for the formation of terrestrial-class planets with considerable amounts of water (Haghighipour \& Raymond 2007).

regions interior to $0.19 q_{b}$ for the formation of terrestrial objects. That means, around a Sun-like star, where the inner edge of the habitable zone is at $\sim 0.9 \mathrm{AU}$, a stellar companion with a perihelion distance smaller than $0.9 / 0.19=4.7 \mathrm{AU}$ would not allow habitable planet formation. In simulations with giant planets, on the other hand, figure 5 shows that terrestrial planets form closer-in. The ratio $a_{\text {out }} / q_{b}$ in these systems is between 0.06 and 0.13. A detailed analysis of our simulations also indicate that the systems, in which habitable planets were formed, have large periastra. The right graph of figure 5 shows this for simulations in a binary with equal-mass Sun-like stars. The circles in this figure represent systems with habitable planets. The numbers on the top of the circles show the mean eccentricity of the giant planet. For comparison, systems with unstable giant 

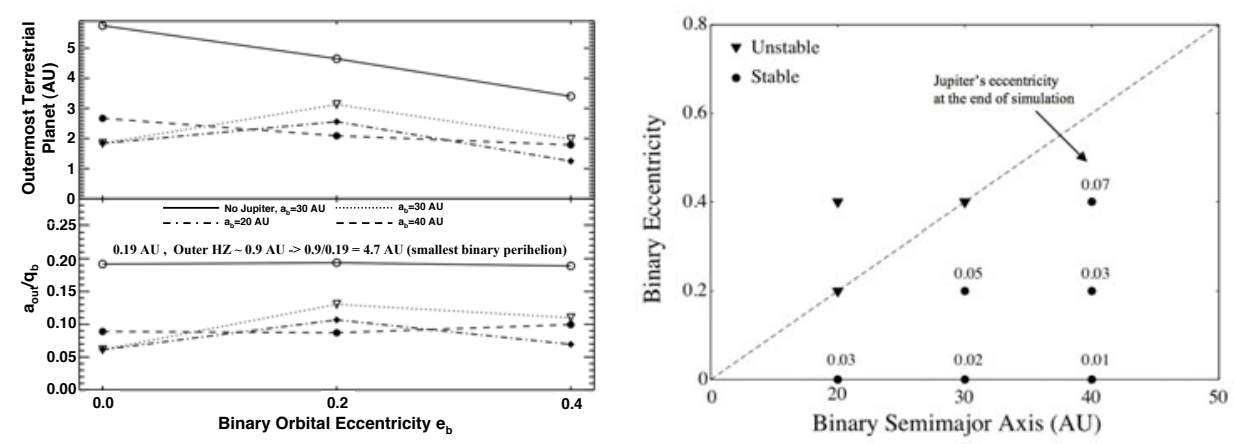

Figure 5. The graph on the left shows the relation between the periastron of an equal-mass binary and the location of its outermost terrestrial planet. The graph on the right shows the region of the $\left(e_{b}, a_{b}\right)$ space for a habitable binary-planetary system (Haghighipour \& Raymond 2007).

planets have also been marked. Since at the beginning of each simulation, the orbit of the giant planet was considered to be circular, a non-zero eccentricity is indicative of the interaction of this body with the secondary star. As shown here, Earth-like objects are formed in systems where the interaction between the giant planet and the secondary star is weak and the average eccentricity of the giant planet is small. That implies, habitable planet formation is more favorable in binaries with moderate to large perihelia, and with giant planets on low eccentricity orbits.

\section{Acknowledgements}

Support by the NASA Astrobiology Institute under Cooperative Agreement NNA04CC08A with the Institute for Astronomy at the University of Hawaii-Manoa is acknowledged.

\section{References}

Abe, Y., Ohtani, E., Okuchi, T., Righter, K., \& Drake, M. 2000, in Origin of the Earth and the Moon, ed. K. Righter \& R. Canup (Tucson: Univ. Arizona Press), 413

Artymowicz, P. \& Lubow, S. H. 1994, ApJ, 421, 651

Dove, A. \& Haghighipour, N. 2006, BAAS, 37, 1284

Els, S. G., Sterzik, M. F., Marchis, F., Pantin, E., Endl, M., \& Kruster, M. 2001, A\& A, 370, L1

Fischer, D. A., Marcy, G. W., Butler, R. P., Vogt, S. S., Laughlin, G., Henry, G. W., Abouav, D., Peek, K. M. G., Wright, J. T., Johnson, J. A., McCarthy, C., \& Isaacson, H. 2008, ApJ, 675,790 .

Haghighipour, N. 2006, ApJ, 644, 543

Haghighipour, N. \& Raymond, S., N. 2007, ApJ, 666, 436

McArthur, B. E., Endl, M., Cochran, W. D., Benedict, G. F., Fischer, D. A., Marcy, G. W., Butler, R. P., Naef, D., Mayor, M., Queloz, D., Udry, S., \& Harrison, T. E. 2004, ApJ, 614, L81

Morbidelli, A., Chambers, J., Lunine, J, I., Petit, J. M., Robert, F., Valsecchi, G., B., \& Cyr, K., E. 2000, Meteorit. Planet. Sci., 35, 1309

Quintana, E. V., Adams, F. C., Lissauer, J. J., \& Chambers, J. E. 2007, ApJ, 660, 807

Raghavan, D., Henry, T. J., Mason, B. D., Subasavage, J. P., Jao, W. C., Beaulieu, T. D., \& Hambly, N. C. 2006, ApJ, 646, 523

Rivera, E. J., Lissauer, J. J., Butler, R. P., Marcy, G. W., Vogt, S. S., Fischer, D. A., Brown,

T. M., Laughlin, G., \& Henry, G. W. 2005, ApJ, 634, 625

Rivera, E. \& Haghighipour, N. 2007, MNRAS, 374, 599

Thébault, P., Marzari, F., Scholl, H., Turrini, D., \& Barbieri, M. 2004, A\& A, 427, 1097

Zucker, S., Mazeh, T., Santos, N. C., Udry, S., \& Mayor, M. 2004, A\&广A, 426, 695 